Monatsschr Kinderheilkd 2012 • 160:333-334

DOI 10.1007/s00112-011-2564-6

Online publiziert: 28. März 2012

(c) Springer-Verlag 2012

L.T. Weber ${ }^{1} \cdot$ F. Zepp ${ }^{2}$

${ }^{1}$ Kinderklinik und Poliklinik, Dr. von Haunersches Kinderspital,

Klinikum, Ludwig-Maximilians-Universität München

${ }^{2}$ Zentrum für Kinder- und Jugendmedizin, Universitätsmedizin Mainz

\title{
Transplantation solider Organe im Kindes- und Jugendalter
}

Splitlebern als weiteren Beitrag zur Erhöhung des Organangebots. Die einzigartige Besonderheit des Organs Leber, nämlich die Fähigkeit zur Regeneration, bietet in speziellen Fällen die Möglichkeit einer temporären partiellen Lebertransplantation (APOLT), die eine auch nur vorübergehende immunsuppressive Therapie erlaubt.

\section{1) Eine optimale \\ Effektivitäts-Toxizitäts-Balance der Immunsuppression \\ ist anzustreben}

In allen Beiträgen wird auf die potenziellen Nebenwirkungen einer immunsuppressiven Therapie eingegangen, die sowohl von deren Intensität als auch deren Dauer abhängen. Die Gefahren einer lang dauernden immunsuppressiven Therapie hinsichtlich opportunistischer Infektionen werden von Huppertz in seinem Beitrag behandelt. Eine wesentliche Herausforderung an die Betreuung transplantierter Patienten stellt daher die beständige Überprüfung der immunsuppressiven Therapie hinsichtlich der individuellen Sicherstellung einer Balance zwischen Effektivität und Toxizität dar. Hierzu stehen neben pharmakokinetischen auch pharmakodynamische und -genetische Methoden eines Drugmonitorings zur Verfügung.

Insbesondere für die Population chronisch kranker Patienten ist Krankheits- prävention durch Impfung vor einer möglichen Transplantation ein Muss. Hierzu nimmt Huppertz in seinem Beitrag ausführlich Stellung.

Die vorliegende Ausgabe der Monatsschrift Kinderheilkunde informiert ausführlich über Indikationen, Durchführung und Nachsorge der Organtransplantation im Kindes- und Jugendalter und fasst den aktuellen Wissensstand übersichtlich zusammen. Die kontinuierliche Verbesserung von Patienten- und Transplantatüberleben bei allen Transplantationen solider Organe bestätigt zweifellos diese Therapieform, ist aber gleichzeitig auch Auftrag, Strategien gegen Langzeitkomplikationen zu entwickeln bzw. zu optimieren. Hierauf weisen Kozlik-Feldmann et al. in ihrem Beitrag zur Transplantation thorakaler Organe hin.

Allerdings verhindern kleine Patientenzahlen nur allzu oft die erfolgreiche Durchführung prospektiver Studien. Wie auch die sorgfältige Erhebung von Verlaufsdaten zur Etablierung von Therapiestandards beitragen kann, beschreiben Tönshoff et al. anhand eines kürzlich etablierten internationalen Registers zur Nierentransplantation im Kindes- und Jugendalter. Nieren werden zahlenmäßig am häufigsten von allen soliden Organen transplantiert, und die präemptive, d. h. ohne vorhergehende Dialysetherapie durchgeführte Nierentransplantation zeigt dabei ein besseres Transplantatüberleben. 
Bei allen positiven Entwicklungen es bleibt keine Zeit, auszuruhen:

- Können wir zukünftig chronischen Transplantatveränderungen besser vorbeugen?

- Werden wir irgendwann immunologische Toleranz induzieren können?

- Wie optimieren wir den Transfer jugendlicher Transplantationspatienten in die Erwachsenenmedizin?

- Wie erfolgreich werden wir in der Vermeidung (kardiovaskulärer) Langzeitkomplikationen sein?

- Wie kann die Compliance jugendlicher Patienten verbessert werden?

Dies ist nur eine Auswahl übergeordneter Fragen, und es bleibt zu hoffen, dass in wirtschaftlich schwierigen Zeiten die ohnehin schon knappen Ressourcen zu ihrer Beantwortung nicht noch weiter reduziert werden.

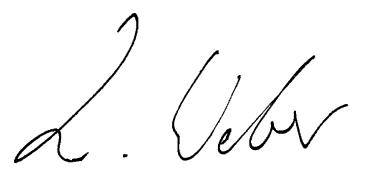

PD Dr. Lutz Weber

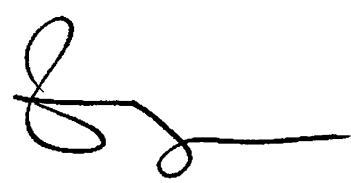

Prof. Dr. Fred Zepp

\section{Korrespondenzadresse}

\section{PD Dr. L.T. Weber}

Kinderklinik und Poliklinik,

Dr. von Haunersches Kinderspital, Klinikum, Ludwig-Maximilians-Universität München Lindwurmstraße 4, 80337 München lutz.weber@med.uni-muenchen.de
Haas NA, Kleideiter U

\section{Kinderkardiologie}

Klinik und Praxis der Herzerkrankungen bei Kindern, Jugendlichen und jungen Erwachsenen

Stuttgart New York: Thieme 2011

502 S., 484 Abb., 110 Tab.

(ISBN 978-3-13-149001-8),

inkl. DVD, 149.00 EUR

"Und noch ein Kinderkardiologie-Buch..." so beginnen die beiden Buchautoren ihr Vorwort, um in weiterer Folge dieses Statement doch zu relativieren. Sie stellen sich selbst als Leiter eines überregionalen Herzkatheterlabors bzw. als Kinderkardiologe einer allgemeinpädiatrischen Klinik vor und nennen als Beweggründe zur Herausgabe dieses Buches u. a. die rasante Entwicklung ihrer Spezialdisziplin und den Wunsch, zahlreiche von ihnen erstellte Ausbildungsskripten in einem Buch zusammenzufassen. Entstanden ist daraus ein 502 Seiten umfassendes Buch mit 484 Abbildungen und 110 Tabellen, das alle Bereiche der Kinderkardiologie abdeckt. Den beiden Autoren gebührt allein schon dafür Hochachtung, dass sie ein Buch dieses Umfangs und dieser Spezialisierung zu zweit zustande gebracht haben.

Das Buch gliedert sich in 5 Kapitel, welche sinnvoll und übersichtlich angeordnet sind. Im Kapitel Grundlagen und Diagnostik werden die in der Kinderkardiologie angewandten Methoden [EKG (Elektrokardiogramm), Echokardiographie, kardiale MRT (Magnetresonanztomographie), Herzkatheter usw.) beschrieben.

Ein kurzes Kapitel Leitsymptome verweist auf die Symptomatik und das Prozedere bei Zyanose, Herzgeräuschen, Thoraxschmerzen und dergleichen.

Im 284 Seiten umfassenden Kapitel Krankheitsbilder werden alle Herzfehler sehr detailliert besprochen, daneben aber auch arterielle Hypertonie, Herzinsuffizienz, syndromale Erkrankungen u. a.

Das Kapitel Therapie beschreibt schließlich allgemein in der Kinderkardiologie zum Einsatz kommende Therapiemaßnahmen wie postoperative Behandlung, Herz-Lungen-Maschine, Herztransplantation und medikamentöse Therapie.

Im Anhang findet man nützliche Tabellen u.

a. mit Normwerten für altersabhängige Klap- pendurchmesser, frequenzkorrigierte QT-Zeit (Abstand vom Beginn der Q-Zacke bis zum Ende der T-Welle) und andere EKG-Intervalle. Der eindeutige Schwerpunkt des Buches liegt auf der Beschreibung der einzelnen Herzfehler. Diese sind gut gegliedert und mit zahlreichen Details von den Grundlagen und der Epidemiologie über Diagnostik bis zur Therapie und Prognose dargestellt und mit gut gelungenen schematischen Farbdarstellungen illustriert. So gelingt es dem Leser, die oft schwierige anatomische und hämodynamische Situation gut nachzuvollziehen. Im beschreibenden Text finden sich mitunter Redundanzen in Form von Wiederholungen, die aber nicht wirklich stören, sondern evtl. sogar zur Festigung des Gelesenen bzw. Gelernten beitragen. Den operativen Korrekturverfahren wird relativ breiter Raum gegeben, wobei die hier beschriebenen Details wohl nur einen beschränkten Leserkreis finden dürften. Auf den ersten Blick mutet es etwas eigenartig an, dass auf Ultraschallbilder in diesem Kapitel fast völlig verzichtet wurde. Allerdings landet man sehr bald bei der beigefügten DVD, auf welcher die wichtigsten Herzfehler "real-time" studiert werden können. Dass Echokardiographien hier zugunsten von Herzkatheterangiographien unterrepräsentiert sind, ist ein kleiner Schönheitsfehler. Mehr und bessere Ultraschallvideos würden wohl den didaktischen Wert der DVD noch erhöhen.

Das Buch gehört wegen der nicht ganz einfachen Materie (insbesondere was anatomische und hämodynamische Gegebenheiten betrifft) sicher nicht zu jenen Büchern, die man in einem Zug durchliest oder studiert. Im Gegenteil erfordert die Lektüre - insbesondere vom nicht täglich kinderkardiologisch Tätigen - viel Konzentration und Durchhaltevermögen. Gleichzeitig aber bietet das Buch eine sehr gute und anschaulich präsentierte Zusammenfassung aller relevanten kinderkardiologischen Erkrankungen und eignet sich ideal zum Nachlesen. Das für etwa 150 EUR im Internethandel erhältliche Buch empfiehlt sich daher für jede pädiatrische Abteilung und Praxis, die (auch) mit kinderkardiologischen Fällen in Kontakt kommt.

Prof. Dr. Reinhold Kerbl, Leoben 